\title{
Determinants of vision function related quality of life of patients undergoing unilateral cataract surgical services in charitable hospitals in India: a multicentre prospective cohort study
}

\author{
Elizabeth Kurian, Pankaj Vishwakarma, Jaikumar Deenadayalan, Amit Mondal, \\ Dhanaji Ranpise, Shobhana Chavan, Prem Kumar S. G.*
}

Mission for Vision, Mumbai, Maharashtra, India

Received: 09 January 2018

Accepted: 03 February 2018

\section{*Correspondence:}

Dr. Prem Kumar SG,

E-mail: pkumar@missionforvision.org.in

Copyright: (C) the author(s), publisher and licensee Medip Academy. This is an open-access article distributed under the terms of the Creative Commons Attribution Non-Commercial License, which permits unrestricted non-commercial use, distribution, and reproduction in any medium, provided the original work is properly cited.

\begin{abstract}
Background: Cataract is the leading cause of avoidable blindness in developing world, including India. The objective of this study is to measure the changes in quality of life (VRQoL) after cataract surgery and identify the predictors of an improvement in these outcomes.

Methods: A multi-center prospective, longitudinal cohort study was conducted. At baseline patients aged $\geq 18$ years with first-eye cataract were interviewed about VRQoL. Six months' later participants were re-interviewed at their residences. Multiple classification analysis (MCA) was performed to assess the variation in the intensities of mean change scores for general function, psychosocial impact and visual function with select factors.

Results: The six-month follow-up rate was $87.3 \%$. There was a significant improvement in visual acuity and VRQoL post-surgery. The mean general function, psychosocial impact and visual function scores were 34.6 (SD 10.9), 10.5 (SD 3.7) and 8.4 (SD 2.2) in the baseline and 15 (SD 5.5), 5 (SD 2.2) and 4.4 (SD 1.6) in the follow-up assessments, respectively. In MCA, patients prescribed spectacles post-surgery $\left(\begin{array}{ll}\beta & 0.137)\end{array}\right)$ and those from rural backgrounds $(\beta$ 0.137 ) had the most impact on general function. Patients with complete follow-up visits had a relatively higher effect on the psychosocial impact $(\beta$ 0.084) whereas patients from rural background and men had the most impact on the visual function scores $(\beta 0.102$ and 0.076$)$ respectively.

Conclusions: Cataract surgery is associated with meaningful improvements in VRQoL in general. The determinants of better VFQoL include regular and complete patient follow-up visits and prescription and provision of spectacles post-surgery.
\end{abstract}

Keywords: Cataract, India, Quality of life, Visual acuity, Avoidable blindness, Spectacles

\section{INTRODUCTION}

Based on the World Health Organization's (WHO) definition of health, quality of life is an integral part of health and well-being and the WHO recommended that vision related quality of life (VRQoL) form part of assessments and evaluations of interventions in eye health. ${ }^{1,2}$ Cataract surgery has a multiplier effect as it not only improves the Quality of Life (QoL) but also prolonging life as people who can see better post-surgery are more likely to get exercise, take their medications and avoid falls. ${ }^{3}$ The age-standardised global prevalence of blindness was reported at $0.48 \%$ in 2015 as against $0.75 \%$ in $1990 .^{4}$ Though this decline is encouraging, cataract continues to remain the main cause of blindness with the vast majority of cataract blind living in low and 
middle income countries (LMIC) and approximately nine million of those blind from cataract live in India. ${ }^{4-7}$ Furthermore, evidence suggests that visual impairment (VI) leads to reduced QoL, poorer general health, lower social status and increased mortality. ${ }^{8-12}$ Cataract surgery is a highly cost-effective intervention and cataract surgical rates have increased considerably over the past decade in many LMICs, including India. ${ }^{13,14}$ The aim of cataract surgery is to improve the vision and thereby the positively impact the quality of lives of people affected. However, the quality of providing cataract surgery is not always optimal, with a considerable proportion of patients still blind or visually impaired after cataract surgery in a LMIC setting. ${ }^{15,16}$

As the Indian population ages, morbidity associated with cataract surgery is expected to rise. In planning medical services for cataract patients, it is essential to include the assessment of post-surgical outcomes not only in terms of visual acuity, but also for patient reported visual functioning and vision related quality of life. There were many studies that attempted to measure variations in the quality of life of patients pre and post-cataract surgery in the past, however, their sample size was limited. ${ }^{1722}$ Of these, majority of studies have used the Visual Function Index (VF-14) to measure VRQoL among cataract patients and the majority were conducted in developed countries. ${ }^{17}$ This instrument has been criticised for not addressing all the visual concerns of cataract patients, ${ }^{18}$ being highly focused on activities that require visual acuity and having ceiling effects. ${ }^{18-20}$ The subsequent National Eye Institute Visual Function Questionnaire (NEI VFQ-25) and the Indian Vision Function Questionnaire (IND-VFQ33) have addresses a wider range of visual concerns, including social and mental outcomes of visual impairment and allows investigation into which specific aspects of VRQoL improve after cataract surgery. ${ }^{21,22}$ Against this background, we assessed the impact of successful first eye cataract surgery on the vision and overall vision related QoL (VRQoL) in India.

\section{METHODS}

\section{Study setting and sample}

Data for this longitudinal study were collected from April 2016 to March 2017 at eighteen private not-for-profit hospitals spread across thirteen Indian states. Study population comprised of adults aged 18 years and above, presenting to the ophthalmology department at these hospitals. They were either walk-in patients or referrals from outreach eye screening camps.

Sample size calculations were powered to detect a $20 \%$ improvement in VRQoL six months after cataract surgery based on previous study findings. ${ }^{8,9,22}$ To detect this improvement required about 200 operated cases in each hospital examined at baseline and follow-up with an alpha of 0.05 and $80 \%$ power. A random-proportional sampling technique was adopted to sample all eligible participants. The sample was proportionately distributed amongst the 18 hospitals based on the annual number of cataract surgeries done during the preceding financial year. Adults who could understand at least one of the following eleven languages- Assamese, Bengali, Gujarati, Hindi, Kannada, Jayantia, Marathi, Oriya, Punjabi, Tamil, Telugu or English were considered eligible for participation.

\section{Data collection: baseline and follow-up}

The data collection happened in two phases; Baseline survey (pre-operative) - initially a day or two prior to initiation of cataract surgery; and Follow-up survey (postoperative) - which took place at the residence of the participants six months after the initial first eye cataract surgery. Standard research protocols were followed during data collection in both the baseline and follow-up surveys in accordance with the Helsinki Declaration. During the baseline assessment, each potential participant was contacted by an interviewer trained in the study procedures with the assistance of the hospital staff. Patients admitted to the ophthalmology inpatient ward for cataract surgery were approached. Before starting the interview, each participant was explained the nature and purpose of the interview and their verbal consent for participation sought. Subsequently, participants were interviewed in an isolated area outside the in-patient ward of the hospital. Average interview time was 45 minutes. Follow-up surveys were undertaken approximately six months from the initial interview. All traced participants were re-examined (using same VA assessment procedures as at baseline) and re-interviewed. Interviews were conducted in respondents' own homes by trained interviewers who were regularly observed by supervisors. The interviewers at baseline and follow up were the same. Interviews were recorded electronically using Patient Related Impact Studying Mechanism (PRISM), a mobile data recording application (CommCare-Dimagi, Inc. Cambridge, MA, USA). ${ }^{23}$

\section{Measures}

The interview documented socio-demographic characteristics of adult including age, sex, education, occupation, place of residence, visual acuity and spectacle use. The pre and post-operative visual acuity and surgery details were documented from the individual patient medical record available with the respective hospitals. Measures of visual acuity (VA) were classified into three broad categories as defined by the World Health Organisation (WHO) as good, borderline or poor. Good outcome was defined as a visual acuity of better than or equal to $6 / 18$ with the available correction; borderline outcome as 6/24-6/60; and poor outcome as $<6 / 60{ }^{24}$ During the baseline survey, VA details were noted from the patient medical records available at the treating hospital. However, during the follow-up survey VA was measured by trained investigators using a 
Snellen tumbling E chart. All measurements were taken in full daylight with available correction. To assess quality of life of adult due to visual impairment, we used the $\mathrm{WHO} /$ prevention of blindness and deafness 20-item visual functioning questionnaire (WHO/PBD VF20) which was in turn adapted from the Indian IND-VFQ33 and proposed by the WHO as a tool for assessing VRQoL in low-income settings. ${ }^{25,26}$ The scale includes 20 items on overall eyesight, visual symptoms, visual functioning and psychosocial well-being, each with a 5-point response option. The WHO/PBD VF20, was translated into the local languages for use by the researchers, and then was back-translated and field-tested to ensure proper readability. The composite score was the sum of all the items and higher scores indicated increased difficulty of visual functionality. If option on visual task was not applicable, it was also mentioned. Two rounds of pretesting of the study instrument were undertaken prior to the start of actual data collection. Based on these exercises, certain definitions were simplified, revisions in local language translation made, and interview techniques improved.

\section{Statistical analysis}

Microsoft office Excel 2013 and SPSS statistical software (Version 20.0, SPSS Science, Chicago, IL) were used to analyze the data. Participants who were not traced or contacted in the follow-up were excluded from all analyses. Descriptive statistical analyses were performed to characterize the participants' socio-demographic, clinical and WHO/PBD VF20 data using Chi-square tests or one-way analysis of variance tests as appropriate. Descriptive statistics for general function, psychosocial impact and visual function sub-scale scores are reported for relevant variables, and independent sample $\mathrm{T}$ test and
ANOVA test were used to assess significance as appropriate. Change scores were computed as a difference between baseline and follow-up scores for the WHO/PBD VF20 and all of its three sub scales. Effect sizes for longitudinal change in VRQoL was calculated as the mean change in scores divided by the standard deviation of the baseline score. Effect sizes of 0.2-0.49 were considered "small," $0.5-0.79$ medium and $\geq 0.8$ large. ${ }^{27}$ Multiple classification analysis (MCA) was performed to assess the variation in the intensities of mean change scores for general function, psychosocial impact and visual function with select factors. Mean change scores for the three sub-scales were considered as dependent variables in the MCA.

\section{RESULTS}

A total of 3,452 adult participants consented to the study and completed baseline assessments.

Response rates at follow-up were generally high; with a total of $3,015(87.3 \%)$ completing the follow-up assessment. Results are presented for participants who completed both baseline and follow-up assessments.

\section{Socio-demographic characteristics}

Table 1 describes the socio-demographic characteristics of the study population. Of the 3,015 participants, half were female $(50.6 \%)$ and aged between 41 and 60 years (49.9\%). The median ages were 63 and 60 for men and women respectively. About three-quarters were currently married (78.2\%). Most participants had no formal education $(57.6 \%)$, and just a little over one-thirds were currently working (38.9\%). A greater majority were rural residents $(81.1 \%)$.

Table 1: Demographic and clinical characteristics of participants who completed baseline and follow-up assessments.

\begin{tabular}{|c|c|c|c|c|}
\hline Variable & Categories & $\begin{array}{l}\text { Total (\%); } \\
\text { N=3,015 }\end{array}$ & $\begin{array}{l}\text { Male (\%); } \\
\mathrm{N}=1,489\end{array}$ & $\begin{array}{l}\text { Female (\%); } \\
\mathrm{N}=1,526\end{array}$ \\
\hline \multirow{4}{*}{ Age } & $\leq 40$ years & $70(2.3)$ & $24(1.6)$ & $46(3)$ \\
\hline & $\overline{4} 1$ to 60 years & $1,505(49.9)$ & $614(41.2)$ & $891(58.4)$ \\
\hline & 61 to 80 years & $1,413(46.9)$ & $830(55.7)$ & $583(38.2)$ \\
\hline & $>80$ years & $27(0.9)$ & $21(1.4)$ & $6(0.4)$ \\
\hline \multirow{3}{*}{ Marital status* } & Never married & $50(1.7)$ & $23(1.5)$ & $27(1.8)$ \\
\hline & Currently married & $2,350(78.2)$ & $1,339(90)$ & $1,011(66.6)$ \\
\hline & Previously married & $606(20.2)$ & $126(8.5)$ & $480(31.6)$ \\
\hline \multirow{4}{*}{ Education } & Illiterate & $1,737(57.6)$ & $587(39.4)$ & $1,150(75.4)$ \\
\hline & Primary schooling & $683(22.7)$ & $459(30.8)$ & $224(14.7)$ \\
\hline & Secondary schooling & $565(18.7)$ & $415(27.9)$ & $150(9.8)$ \\
\hline & College or more & $30(1)$ & $28(1.9)$ & $2(0.1)$ \\
\hline \multirow{2}{*}{ Place of residence } & Urban & $570(18.9)$ & $266(17.9)$ & $304(19.9)$ \\
\hline & Rural & $2,445(81.1)$ & $1,223(82.1)$ & $1,222(80.1)$ \\
\hline \multirow{2}{*}{$\begin{array}{l}\text { Job other than working on } \\
\text { own field }\end{array}$} & Yes & $1,173(38.9)$ & $650(43.7)$ & $523(34.3)$ \\
\hline & No & $1,842(61.1)$ & $839(56.3)$ & $1,003(65.7)$ \\
\hline
\end{tabular}




\begin{tabular}{|c|c|c|c|c|}
\hline Variable & Categories & $\begin{array}{l}\text { Total }(\%) \\
\text { N=3,015 }\end{array}$ & $\begin{array}{l}\text { Male (\%); } \\
\mathrm{N}=1,489\end{array}$ & $\begin{array}{l}\text { Female }(\%) \\
N=1,526\end{array}$ \\
\hline \multirow{4}{*}{ Own social security cards } & BPL card & $2,557(84.8)$ & $1239(83.2)$ & $1,318(86.4)$ \\
\hline & GHI card & $184(6.1)$ & $122(8.2)$ & $62(4.1)$ \\
\hline & Both & $22(0.7)$ & $13(0.9)$ & $9(0.6)$ \\
\hline & None & $252(8.4)$ & $115(7.7)$ & $137(9)$ \\
\hline \multirow{2}{*}{ Surgery done on the eye } & Right eye & $1,640(54.4)$ & $794(53.3)$ & $846(55.4)$ \\
\hline & Left eye & $1,375(45.6)$ & 695 (46.7) & $680(44.6)$ \\
\hline \multirow{3}{*}{$\begin{array}{l}\text { Baseline visual acuity in } \\
\text { operated eye }\end{array}$} & Good & $53(1.8)$ & $26(1.7)$ & $27(1.8)$ \\
\hline & Borderline & 964 (32) & $483(32.4)$ & 481 (31.5) \\
\hline & Poor & $1,998(66.3)$ & $980(65.8)$ & $1,018(66.7)$ \\
\hline \multirow{3}{*}{$\begin{array}{l}\text { Follow-up visual acuity in } \\
\text { operated eye }\end{array}$} & Good & $2,688(89.2)$ & $1,325(89)$ & $1,363(89.3)$ \\
\hline & Borderline & $223(7.4)$ & $103(6.9)$ & $120(7.9)$ \\
\hline & Poor & $104(3.4)$ & $61(4.1)$ & $43(2.8)$ \\
\hline \multirow{2}{*}{$\begin{array}{l}\text { Complete post-operative } \\
\text { review attended }\end{array}$} & Yes & $615(20.4)$ & $283(19)$ & $332(21.8)$ \\
\hline & No & $2,400(79.6)$ & $1,206(81)$ & 1,194 (78.2) \\
\hline \multirow{2}{*}{$\begin{array}{l}\text { Spectacles prescribed post- } \\
\text { surgery }\end{array}$} & Yes & $723(24)$ & $363(24.4)$ & $360(23.6)$ \\
\hline & No & $2,292(76)$ & $1,126(75.6)$ & $1,166(76.4)$ \\
\hline \multirow{2}{*}{$\begin{array}{l}\text { Ocular complaints post- } \\
\text { surgery }\end{array}$} & Yes & $562(18.6)$ & $248(16.7)$ & 314 (20.6) \\
\hline & No & $2,453(81.4)$ & $1,241(83.3)$ & $1,212(79.4)$ \\
\hline
\end{tabular}

BPL - Below poverty line; GHI - Government health insurance; * Data missing for 9 cases.

\section{Clinical characteristics and visual acuity}

All participants had cataracts and a little over half reported surgery in the right eye $(54.4 \%)$. The grades of visual acuity of the operated eye at six months and before surgery were compared for unilateral eye cataract surgeries (Table 1). Considerable improvement was noted in the vision six months after cataract surgery among adults operated for cataract. Prior to the cataract surgery, majority $(66.3 \%)$ had a poor VA reading, followed by those with a borderline VA (32\%). However, following the surgery, an overwhelming number of them had good VA $(89.2 \%)$. About $20 \%$ patients had completed full post-operative review and $24 \%$ were provided with spectacles post-surgery.

\section{VRQoL - WHO/PBD VF20}

The overall mean VRQoL score was 56.6 (Standard Deviation, SD 15.5) and 26.4 (SD 8.8) in the baseline and follow-up respectively. The mean general function, psychosocial impact and visual function scores were 34.6 (SD 10.9), 10.5 (SD 3.7) and 8.4 (SD 2.2) in the baseline and 15 (SD 5.5), 5 (SD 2.2) and 4.4 (SD 1.6) in the follow-up assessments, respectively. Figure 1 shows the distribution of overall mean scores for VRQoL using WHO/PBD VF20 for the baseline and follow-up assessments. The distribution of overall VRQoL score was clustered above the mean level in the baseline as against lower than the mean level during the follow-up assessment, signifying a significant improvement in the quality of life of patients' post-surgery $(p<0.001$; Figure $1)$.

The distribution of the overall VRQoL score by grades of VA are presented in Figure 2.

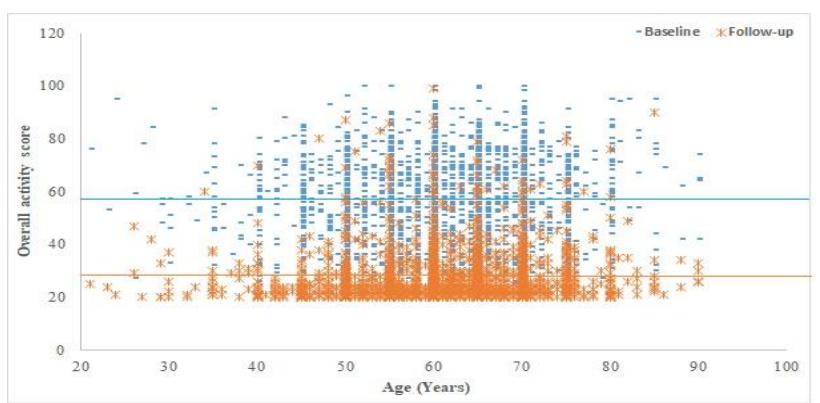

Figure 1: Distribution of overall activity score by age before and after cataract surgery amongst adult patients in India.

A statistically significant association was seen in overall VRQoL scores and improvements in VA before and after cataract surgery as the median QoL scores exhibit a sharp decline in the follow-up scenario $(\mathrm{p}<0.001$; Figure 2$)$.

A good baseline VA and those who were prescribed spectacles post-surgery were consistently associated with greater improvements in general functioning, psychosocial impact and visual function scores after adjustment for baseline score (Table 2). Furthermore, mean change score for general functioning was significantly associated with men $(\mathrm{p}<0.001)$, those who had an education higher that college or more $(\mathrm{p}=0.002)$ and those who were prescribed spectacles $(p<0.001)$. Patients residing in urban areas $(\mathrm{p}=0.002)$, those who had jobs other than working on own farms $(\mathrm{p}=0.007)$ and those who completed full post-operative follow-up reviews $(\mathrm{p}=0.04)$ had exhibited significant association with psychosocial impact scores. Male patients $(\mathrm{p}<0.001)$ and those residing in rural areas $(p<0.001)$ had a significant association with visual function scores (Table 2). 


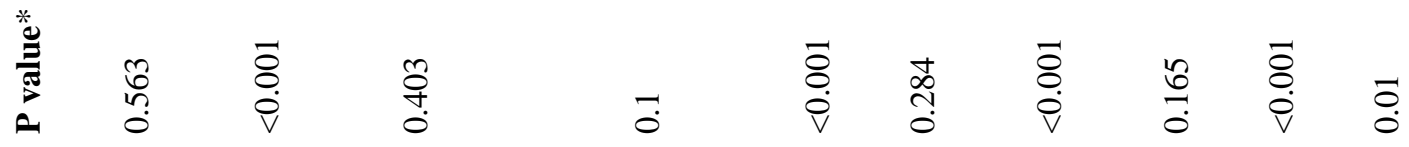

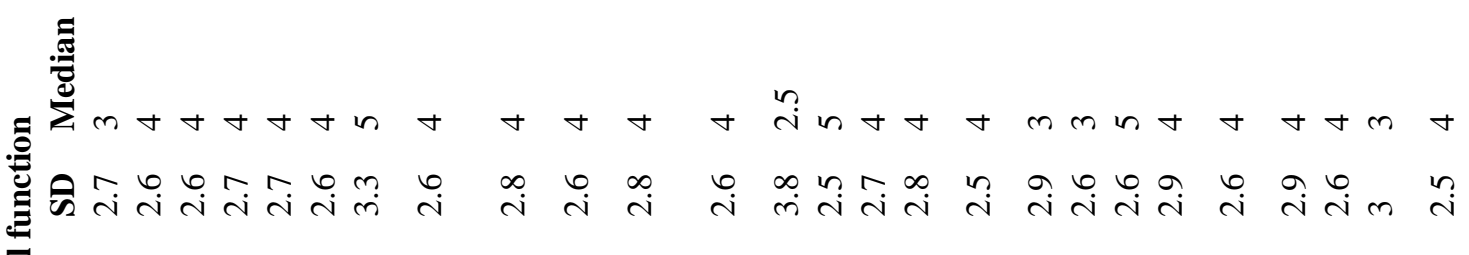

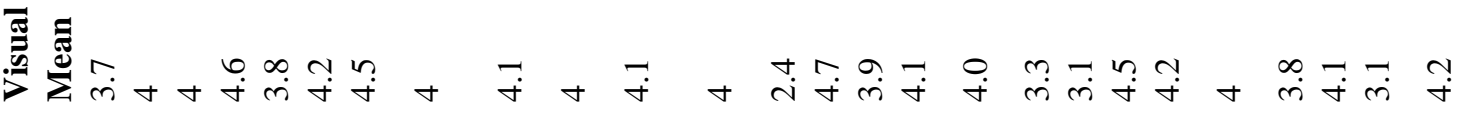

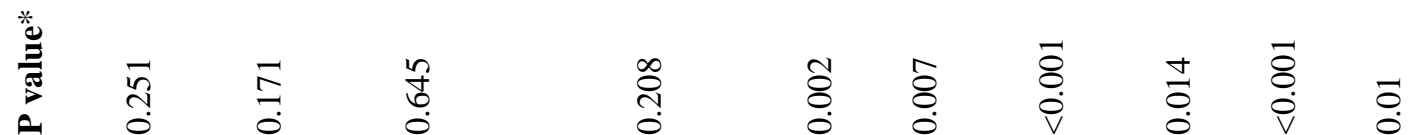

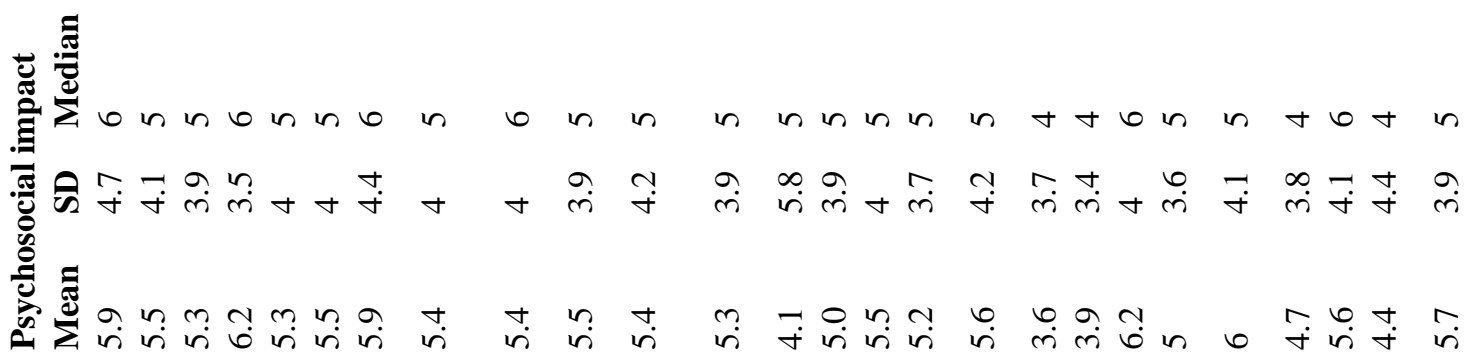
$\frac{0.00}{n}$

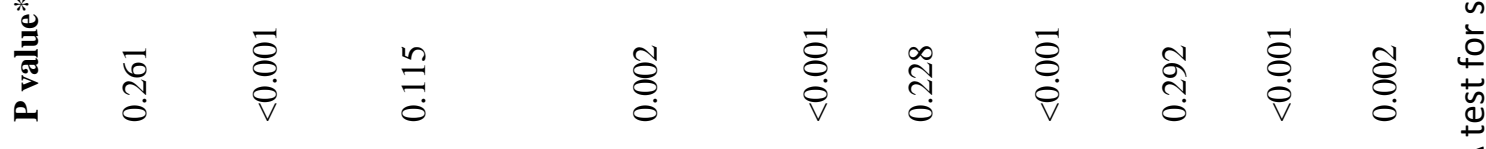
๘ 昜

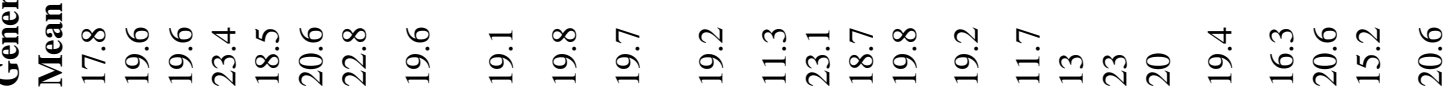

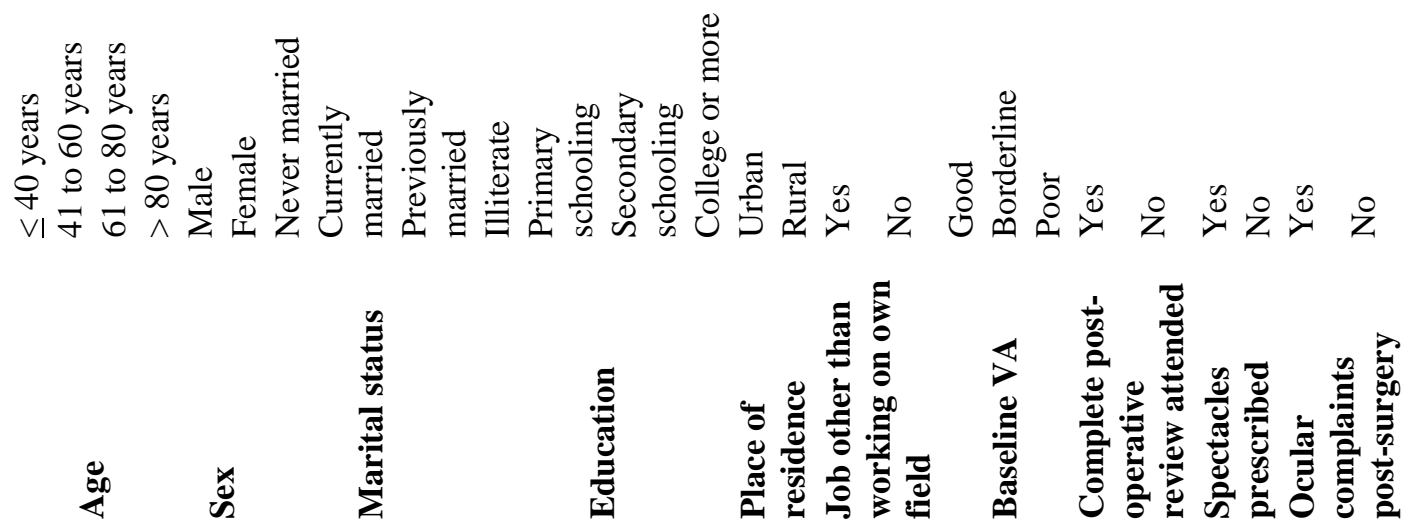


Table 3: Contribution of WHO/PBD VF20 items towards the burden of quality of life among the adult patients operated for cataract in India.

\begin{tabular}{|c|c|c|c|c|c|c|c|c|c|c|}
\hline & \multicolumn{5}{|c|}{ Baseline $(\mathrm{N}=3,015)$} & \multicolumn{5}{|c|}{ Follow-up $(\mathrm{N}=3,015)$} \\
\hline & $\begin{array}{l}\text { Option } \\
1\end{array}$ & $\begin{array}{l}\text { Option } \\
2\end{array}$ & $\begin{array}{l}\text { Option } \\
3\end{array}$ & $\begin{array}{l}\text { Option } \\
4\end{array}$ & $\begin{array}{l}\text { Option } \\
5\end{array}$ & $\begin{array}{l}\text { Option } \\
1\end{array}$ & $\begin{array}{l}\text { Option } \\
2\end{array}$ & $\begin{array}{l}\text { Option } \\
3\end{array}$ & $\begin{array}{l}\text { Option } \\
4\end{array}$ & $\begin{array}{l}\text { Option } \\
5\end{array}$ \\
\hline \multirow{2}{*}{$\begin{array}{l}\text { Overall, how would you rate your } \\
\text { eyesight using both eyes - with glasses } \\
\text { or contact lenses if you wear them?* }\end{array}$} & 37 & 703 & 1,278 & 882 & 115 & 1,060 & 1,482 & 226 & 232 & 15 \\
\hline & $1.2 \%$ & $23.3 \%$ & $42.4 \%$ & $29.3 \%$ & $3.8 \%$ & $35.2 \%$ & $49.2 \%$ & $7.5 \%$ & $7.7 \%$ & $0.5 \%$ \\
\hline \multicolumn{11}{|l|}{ General function } \\
\hline \multirow{2}{*}{$\begin{array}{l}\text { Because of your eyesight, how much } \\
\text { difficulty do you have in going down } \\
\text { steps or stairs?† }\end{array}$} & 383 & 644 & 1,005 & 805 & 178 & 2,434 & 430 & 112 & 34 & 5 \\
\hline & $12.7 \%$ & $21.4 \%$ & $33.3 \%$ & $26.7 \%$ & $5.9 \%$ & $80.7 \%$ & $14.3 \%$ & $3.7 \%$ & $1.1 \%$ & $0.2 \%$ \\
\hline \multirow{2}{*}{$\begin{array}{l}\text { How much difficulty do you have in } \\
\text { noticing obstacles while you are } \\
\text { walking alone (e.g. animals or } \\
\text { vehicles)?† }\end{array}$} & 360 & 748 & 1,088 & 702 & 117 & 2,385 & 485 & 99 & 39 & 7 \\
\hline & $11.9 \%$ & $24.8 \%$ & $36.1 \%$ & $23.3 \%$ & $3.9 \%$ & $79.1 \%$ & $16.1 \%$ & $3.3 \%$ & $1.3 \%$ & $0.2 \%$ \\
\hline \multirow{2}{*}{$\begin{array}{l}\text { Because of your eyesight, how much } \\
\text { difficulty do you have in searching for } \\
\text { something on a crowed shelf? } \uparrow\end{array}$} & 459 & 761 & 1,079 & 579 & 137 & 2,472 & 419 & 91 & 28 & 5 \\
\hline & $15.2 \%$ & $25.2 \%$ & $35.8 \%$ & $19.2 \%$ & $4.5 \%$ & $82.0 \%$ & $13.9 \%$ & $3.0 \%$ & $0.9 \%$ & $0.2 \%$ \\
\hline \multirow{2}{*}{$\begin{array}{l}\text { How much difficulty do you have in } \\
\text { seeing differences in colours? } \dagger\end{array}$} & 780 & 650 & 727 & 636 & 222 & 2,466 & 422 & 98 & 20 & 9 \\
\hline & $25.9 \%$ & $21.6 \%$ & $24.1 \%$ & $21.1 \%$ & $7.4 \%$ & $81.8 \%$ & $14.0 \%$ & $3.3 \%$ & $0.7 \%$ & $0.3 \%$ \\
\hline \multirow{2}{*}{$\begin{array}{l}\text { Because of your eyesight, how much } \\
\text { difficulty do you have in recognizing } \\
\text { the face of a person standing near you? } \uparrow\end{array}$} & 802 & 963 & 794 & 377 & 79 & 2,831 & 125 & 42 & 14 & 3 \\
\hline & $26.6 \%$ & $31.9 \%$ & $26.3 \%$ & $12.5 \%$ & $2.6 \%$ & $93.9 \%$ & $4.1 \%$ & $1.4 \%$ & $0.5 \%$ & $0.1 \%$ \\
\hline \multirow{2}{*}{$\begin{array}{l}\text { How much difficulty do you have in } \\
\text { seeing the level in a container when } \\
\text { pouring? } \dagger\end{array}$} & 731 & 634 & 857 & 614 & 179 & 2,597 & 321 & 68 & 23 & 6 \\
\hline & $24.2 \%$ & $21.0 \%$ & $28.4 \%$ & $20.4 \%$ & $5.9 \%$ & $86.1 \%$ & $10.6 \%$ & $2.3 \%$ & $0.8 \%$ & $0.2 \%$ \\
\hline \multirow{2}{*}{$\begin{array}{l}\text { Because of your eyesight, how much } \\
\text { difficulty do you have in going to } \\
\text { activities outside of the house (e.g. } \\
\text { sporting events, shopping, religious } \\
\text { events)?† }\end{array}$} & 476 & 509 & 1,058 & 656 & 316 & 2,454 & 389 & 104 & 28 & 40 \\
\hline & $15.8 \%$ & $16.9 \%$ & $35.1 \%$ & $21.8 \%$ & $10.5 \%$ & $81.4 \%$ & $12.9 \%$ & $3.4 \%$ & $0.9 \%$ & $1.3 \%$ \\
\hline \multirow{2}{*}{$\begin{array}{l}\text { Because of your eyesight, how much } \\
\text { difficulty do you have in recognizing } \\
\text { people you know from a distance of } 20 \\
\text { meters?† }\end{array}$} & 48 & 112 & 807 & 1,064 & 984 & 1,969 & 695 & 273 & 48 & 30 \\
\hline & $1.6 \%$ & $3.7 \%$ & $26.8 \%$ & $35.3 \%$ & $32.6 \%$ & $65.3 \%$ & $23.1 \%$ & $9.1 \%$ & $1.6 \%$ & $1.0 \%$ \\
\hline \multirow{2}{*}{$\begin{array}{l}\text { How much difficulty do you have in } \\
\text { seeing close objects (e.g. making out } \\
\text { differences in coins or notes, reading } \\
\text { newsprint)?† }\end{array}$} & 528 & 819 & 926 & 557 & 185 & 2,489 & 400 & 90 & 24 & 12 \\
\hline & $17.5 \%$ & $27.2 \%$ & $30.7 \%$ & $18.5 \%$ & $6.1 \%$ & $82.6 \%$ & $13.3 \%$ & $3.0 \%$ & $0.8 \%$ & $0.4 \%$ \\
\hline \multirow{2}{*}{$\begin{array}{l}\text { How much difficulty do you have in } \\
\text { seeing irregularities in the path when } \\
\text { walking (e.g. potholes)?† }\end{array}$} & 191 & 709 & 1,110 & 775 & 230 & 2,331 & 553 & 95 & 29 & 7 \\
\hline & $6.3 \%$ & $23.5 \%$ & $36.8 \%$ & $25.7 \%$ & $7.6 \%$ & $77.3 \%$ & $18.3 \%$ & $3.2 \%$ & $1.0 \%$ & $0.2 \%$ \\
\hline \multirow{2}{*}{$\begin{array}{l}\text { How much difficulty do you have in } \\
\text { doing activities that require you to see } \\
\text { well close up (e.g. sewing, using hand } \\
\text { tools)?† }\end{array}$} & 217 & 638 & 682 & 813 & 665 & 2,442 & 394 & 127 & 37 & 15 \\
\hline & $7.2 \%$ & $21.2 \%$ & $22.6 \%$ & $27.0 \%$ & $22.1 \%$ & $81.0 \%$ & $13.1 \%$ & $4.2 \%$ & $1.2 \%$ & $0.5 \%$ \\
\hline \multirow{2}{*}{$\begin{array}{l}\text { Because of your eyesight, how much } \\
\text { difficulty do you have in carrying out } \\
\text { your usual work?† } \dagger\end{array}$} & 740 & 628 & 993 & 538 & 116 & 2,599 & 329 & 61 & 26 & 0 \\
\hline & $24.5 \%$ & $20.8 \%$ & $32.9 \%$ & $17.8 \%$ & $3.8 \%$ & $86.2 \%$ & $10.9 \%$ & $2.0 \%$ & $0.9 \%$ & $0.0 \%$ \\
\hline \multicolumn{11}{|l|}{ Psychosocial impact } \\
\hline \multirow{2}{*}{$\begin{array}{l}\text { Because of your eyesight, how often } \\
\text { have you been hesitant to participate in } \\
\text { social functions? } \$\end{array}$} & 762 & 604 & 930 & 509 & 210 & 2,452 & 403 & 111 & 37 & 12 \\
\hline & $25.3 \%$ & $20.0 \%$ & $30.8 \%$ & $16.9 \%$ & $7.0 \%$ & $81.3 \%$ & $13.4 \%$ & $3.7 \%$ & $1.2 \%$ & $0.4 \%$ \\
\hline \multirow{2}{*}{$\begin{array}{l}\text { Because of your eyesight, how often } \\
\text { have you found that you are ashamed or } \\
\text { embarrassed?t }\end{array}$} & 679 & 951 & 878 & 327 & 180 & 2,583 & 300 & 92 & 30 & 10 \\
\hline & $22.5 \%$ & $31.5 \%$ & $29.1 \%$ & $10.8 \%$ & $6.0 \%$ & $85.7 \%$ & $10.0 \%$ & $3.1 \%$ & $1.0 \%$ & $0.3 \%$ \\
\hline \multirow{2}{*}{$\begin{array}{l}\text { Because of your eyesight, how often } \\
\text { have you felt that you are a burden on } \\
\text { others? } \$\end{array}$} & 913 & 901 & 723 & 337 & 141 & 2,567 & 327 & 96 & 19 & 6 \\
\hline & $30.3 \%$ & $29.9 \%$ & $24.0 \%$ & $11.2 \%$ & $4.7 \%$ & $85.1 \%$ & $10.8 \%$ & $3.2 \%$ & $0.6 \%$ & $0.2 \%$ \\
\hline \multirow{2}{*}{$\begin{array}{l}\text { Because of your eyesight, how often do } \\
\text { you worry that you may lose your } \\
\text { remaining eyesight? }\end{array}$} & 351 & 594 & 901 & 757 & 412 & 2,323 & 374 & 206 & 84 & 28 \\
\hline & $11.6 \%$ & $19.7 \%$ & $29.9 \%$ & $25.1 \%$ & $13.7 \%$ & $77.0 \%$ & $12.4 \%$ & $6.8 \%$ & $2.8 \%$ & $0.9 \%$ \\
\hline
\end{tabular}




\section{Visual function}

\begin{tabular}{|c|c|c|c|c|c|c|c|c|c|c|}
\hline \multirow{2}{*}{$\begin{array}{l}\text { How much pain or discomfort do you } \\
\text { have in your eyes (e.g. burning, itching, } \\
\text { aching) }{ }^{\dagger}\end{array}$} & 1,479 & 898 & 458 & 153 & 27 & 2,303 & 605 & 84 & 20 & 3 \\
\hline & $49.1 \%$ & $29.8 \%$ & $15.2 \%$ & $5.1 \%$ & $0.9 \%$ & $76.4 \%$ & $20.1 \%$ & $2.8 \%$ & $0.7 \%$ & $0.1 \%$ \\
\hline \multirow{2}{*}{$\begin{array}{l}\text { How much difficulty do you have in } \\
\text { seeing because of glare from bright } \\
\text { lights? } \dagger\end{array}$} & 57 & 587 & 1,153 & 911 & 307 & 1,683 & 929 & 307 & 88 & 8 \\
\hline & $1.9 \%$ & $19.5 \%$ & $38.2 \%$ & $30.2 \%$ & $10.2 \%$ & $55.8 \%$ & $30.8 \%$ & $10.2 \%$ & $2.9 \%$ & $0.3 \%$ \\
\hline \multirow{2}{*}{$\begin{array}{l}\text { How much difficulty do you have in } \\
\text { seeing when coming inside after being } \\
\text { in bright sunlight? } \dagger\end{array}$} & 58 & 542 & 1,107 & 815 & 493 & 1,747 & 990 & 227 & 39 & 12 \\
\hline & $1.9 \%$ & $18.0 \%$ & $36.7 \%$ & $27.0 \%$ & $16.4 \%$ & $57.9 \%$ & $32.8 \%$ & $7.5 \%$ & $1.3 \%$ & $0.4 \%$ \\
\hline
\end{tabular}
in bright sunlight? $\dagger$

*Option 1: very good; option 2: good; option 3: moderate; option 4: bad; option 5: very bad; †Option 1: none; option 2: mild; option 3: moderate; option 4: severe; option 5: extreme; † Option 1: never; option 2: rarely; option 3: sometimes; option 4: often; option 5: very often.

Table 4: Comparison of baseline and follow-up VRQoL scores and its effect sizes among adult patients in India.

\begin{tabular}{|llllllll|}
\hline \multirow{2}{*}{$\begin{array}{l}\text { WHO/PBD VF20 and its sub- } \\
\text { scales }\end{array}$} & Baseline & \multicolumn{3}{c}{ Follow-up } & \multicolumn{4}{c|}{ Change from BL to } & \multirow{2}{*}{ Effect size* } \\
\cline { 2 - 7 } & Mean & SD & Mean & SD & Mean & SD & \\
\hline Overall activity score & 56.6 & 15.5 & 26.4 & 8.8 & 30.2 & 17.8 & 1.9 \\
\hline General function score & 34.6 & 10.9 & 15 & 5.5 & 19.6 & 12.3 & 1.8 \\
\hline Psychosocial impact score & 10.5 & 3.7 & 5 & 2.2 & 5.4 & 4 & 1.5 \\
\hline Visual function score & 8.4 & 2.2 & 4.4 & 1.6 & 4.0 & 2.7 & 1.8 \\
\hline
\end{tabular}

SD - Standard deviation; BL - Baseline; FU - Follow-up; *Calculated as the mean change score from pre-surgery to post-surgery, divided by the standard deviation of the pre-surgery score.

Table 5: Multiple classification analysis for effect of socio-demographic and clinical characteristics on the level of net VFQoL scores in adult patients operated for cataract in India.

\begin{tabular}{|c|c|c|c|c|c|c|c|c|c|c|c|}
\hline \multirow{3}{*}{ Variable } & \multirow{3}{*}{ Categories } & \multirow{3}{*}{$\mathrm{N}=3,006$} & \multicolumn{9}{|c|}{ Adjusted predicted mean } \\
\hline & & & \multicolumn{3}{|c|}{$\begin{array}{l}\text { Change score - general } \\
\text { function }\end{array}$} & \multicolumn{3}{|c|}{$\begin{array}{l}\text { Change score - } \\
\text { Psychosocial impact }\end{array}$} & \multicolumn{3}{|c|}{$\begin{array}{l}\text { Change score - Visual } \\
\text { function }\end{array}$} \\
\hline & & & Mean & Beta $\beta$ & $P$ value & Mean & Beta $\beta$ & $P$ value & Mean & Beta $\beta$ & P value \\
\hline \multirow{4}{*}{ Age } & $<40$ years & 70 & 17 & \multirow{4}{*}{0.044} & \multirow{4}{*}{0.081} & 5.9 & \multirow{4}{*}{0.039} & \multirow{4}{*}{0.191} & 3.6 & \multirow{4}{*}{0.032} & \multirow{4}{*}{0.370} \\
\hline & 41 to 60 years & 1,497 & 19.4 & & & 5.5 & & & 4 & & \\
\hline & 61 to 80 years & 1,412 & 19.8 & & & 5.3 & & & 4.1 & & \\
\hline & $>80$ years & 27 & 23 & & & 5.9 & & & 4.5 & & \\
\hline \multirow{2}{*}{ Sex } & Male & 1,488 & 18.4 & \multirow{2}{*}{0.091} & \multirow{2}{*}{$<0.001$} & 5.4 & \multirow{2}{*}{0.012} & \multirow{2}{*}{0.562} & 3.8 & \multirow{2}{*}{0.076} & \multirow{2}{*}{$<0.001$} \\
\hline & Female & 1,518 & 20.7 & & & 5.5 & & & 4.2 & & \\
\hline \multirow{3}{*}{ Marital status* } & Never married & 50 & 20.7 & \multirow{3}{*}{0.036} & \multirow{3}{*}{0.113} & 5.9 & \multirow{3}{*}{0.016} & \multirow{3}{*}{0.673} & 4.1 & \multirow{3}{*}{0.010} & \multirow{3}{*}{0.852} \\
\hline & Currently married & 2,350 & 19.8 & & & 5.4 & & & 4 & & \\
\hline & Previously married & 606 & 18.7 & & & 5.5 & & & 4 & & \\
\hline \multirow{4}{*}{ Education } & Illiterate & 1,730 & 19.5 & & & 5.4 & & & 4 & & \\
\hline & Primary schooling & 681 & 20.1 & 0063 & 0002 & 5.5 & 0026 & 0550 & 4.1 & 0062 & 0007 \\
\hline & Secondary schooling & 565 & 19.6 & 0.003 & 0.002 & 5.4 & 0.020 & 0.550 & 4.1 & 0.002 & 0.001 \\
\hline & College or more & 30 & 12.3 & & & 4.4 & & & 2.5 & & \\
\hline Place of recidence & Urban & 568 & 22.2 & 0103 & $<0001$ & 4.7 & 0040 & $<0001$ & 4.6 & 0102 & $<0001$ \\
\hline Place or resiaence & Rural & 2,438 & 18.9 & 0.103 & $<0.001$ & 5.6 & 0.040 & -0.001 & 3.9 & 0.102 & $<0.001$ \\
\hline Job other than & Yes & 1,835 & 19.4 & & & 5.2 & & & 4 & & \\
\hline $\begin{array}{l}\text { working on own } \\
\text { field }\end{array}$ & No & 1,171 & 19.7 & 0.012 & 0.474 & 5.7 & 0.043 & 0.017 & 4 & 0.012 & 0.497 \\
\hline & Good & 53 & 12.6 & & & 3.7 & & & 3.3 & & \\
\hline Baseline VA & Borderline & 964 & 13.4 & 0.359 & $<0.001$ & 3.7 & 0.280 & $<0.001$ & 3.2 & 0.220 & $<0.001$ \\
\hline & Poor & 1,998 & 22.7 & & & 6.2 & & & 4.4 & & \\
\hline Complete post- & Yes & 2,811 & 21.4 & & & 5 & & & 4.2 & & \\
\hline $\begin{array}{l}\text { operative review } \\
\text { attended }\end{array}$ & No & 204 & 19.1 & 0.078 & $<0.001$ & 6 & 0.084 & 0.004 & 4 & 0.039 & 0.050 \\
\hline Spectacles & Yes & 722 & 16.5 & & & 5 & & & 3.9 & & \\
\hline $\begin{array}{l}\text { prescribed post- } \\
\text { surgery }\end{array}$ & No & 2,284 & 20.5 & 0.137 & $<0.001$ & 5.6 & 0.060 & 0.002 & 4.1 & 0.034 & 0.091 \\
\hline & Full model & 3,006 & & 0.190 & $<0.001$ & & 0.096 & $<0.001$ & & 0.077 & $<0.001$ \\
\hline
\end{tabular}

VA - Visual acuity; $* 9$ cases for which data were missing were excluded from the model. 

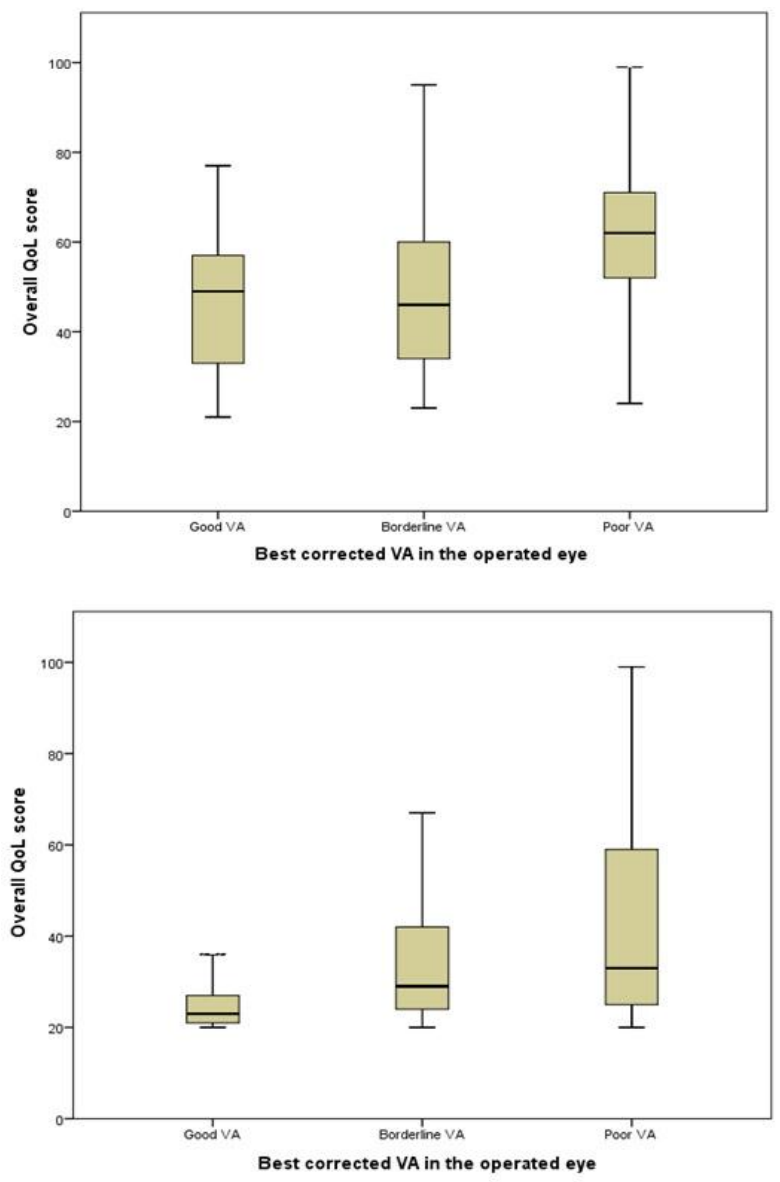

Figure 2: Distribution of the overall QoL score by grades of visual activity among adults before (upper) and after (lower) cataract surgery in India.

QoL: Quality of Life; VA: Visual Acuity.

Table 3 shows distribution of items on the WHO/PBD VF20 scale for the study participants in the baseline and follow-up assessments. The overall eye sight as perceived by the participants was 'moderate' $(42.4 \%)$ as against 'very good and good' (84.3\%) during baseline and follow-up respectively. In the baseline scenario, the general function items that were reported by the majority were: difficulty in recognizing people you know from a distance of 20 meters (severe and extreme combined, $67.9 \%$ ), difficulty seeing close objects (severe and extreme combined, 49\%), difficulty seeing irregularities in the path when walking (severe and extreme combined, $33.3 \%$ ) and difficulty going down steps or stairs (severe and extreme combined, $32.6 \%$ ). However, the majority of the scores post-surgery for these items were (very good and good combined) $88.4 \%, 94.1 \%, 95.7 \%$ and $95 \%$ respectively, suggesting significant improvement. Similarly, the psychosocial impact scores in the baseline that were reported by majority was: how often do you worry that you may lose your remaining eyesight (often and very often combined, $38.8 \%$ ) as against $77 \%$ who reported 'never' six months' post-surgery. With regards to the visual function, the major item towards the score contribution in the baseline were: difficulty do you have in seeing when coming inside after being in bright sunlight (severe and extreme combined, 43.3\%) and difficulty do you have in seeing because of glare from bright lights (severe and extreme combined, 40.4\%) as against $57.9 \%$ and $55.8 \%$ who reported 'none' during the follow-up assessment (Table 3).

WHO/PBD VF20 scores showed improvements after surgery, indicating good overall VFQoL. The effect sizes are large (all exceed 1.00) across the three sub-scales suggestive of improved quality of life of patients' postsurgery (Table 4).

Table 5 shows the MCA for the adjusted predicted mean change scores for general function, psychosocial impact and visual function scores. As expected, a good baseline VA had the highest effect on the intensity of general function, psychosocial impact and visual function scores with a beta value of $0.359,0.280$ and 0.220 respectively. patients who were prescribed spectacles post-surgery $(\beta$ $0.137)$ and those from rural backgrounds $(\beta$ 0.137) had the most impact on general function. Regular and complete patient follow-up visits had a relatively higher effect on the psychosocial impact $(\beta$ 0.084) whereas patients from rural background and men had the most impact on the visual function scores ( $\beta 0.102$ and 0.076$)$ respectively.

\section{DISCUSSION}

This multi-center study found that six months after cataract surgery there were large, significant improvements in visual outcomes, reduced difficulty undertaking everyday activities (general functioning), reduced frequency of negative psychosocial experiences associated with vision and reduced difficulty related to visual functions such as pain or discomfort in the eye or adaptations to varying light conditions. The improvements in VRQoL (measured through WHO/PBD VF20) observed in this study are consistent with a previous study in India and rest of the world which found similarly large effect sizes for VRQoL following cataract surgery. ${ }^{26,28-31}$

This study found that there was a significant association between change in VRQoL and baseline VA with about three-quarters rating vision to be moderate or bad during baseline as against $84 \%$ rating it to be very good or good six-months post-surgery. We also found a significant higher proportion of patients with rural backgrounds, predominantly male patients and those who were prescribed and provided spectacles post-surgery had lower mean change scores for both general and visual functions indicating substantial improvements in quality of life. A recent large population based study amongst adults in southern India reiterated that correction of refractive errors is required to provide good visual recovery and achieve the benefit of cataract surgery. ${ }^{32}$ Most of these free-surgeries in rural areas are done through outreach programs as part of the National 
Program for Control of Blindness (NPCB) activity where they are transported to the base hospital for surgeries and a few given one-time free spectacles six weeks after cataract surgery. Though it is mandatory to prescribe and provide spectacles free of cost to post-surgery to poor patients, we found that just about a quarter of patients were prescribed and provided spectacles. ${ }^{33}$ The current practice with regard to spectacle provision at all of these private nor-for-profit hospitals include providing free spectacles only to those patients who have undergone bilateral cataract surgeries. In the light of the findings of this study, it is recommended that efforts should be made to prescribe and provide spectacles to all the patients requiring spectacles post-surgery even if they were unilateral first-eye cataract patients, as this would substantially enhance the overall quality of life.

The results also confirm that patients in the urban settings, those who had a complete sequence of regular follow-up visits to the hospital post-surgery and those who were prescribed spectacles had better psychosocial outcomes. Generally, patients are expected to have at least two visits to the hospital following cataract surgery - initially one to two weeks and later at thirty to forty days, to monitor the recovery and assess the visual outcomes post-surgery. ${ }^{33}$ Better medical advices and counseling during these follow-up visits might have had a direct psychological benefit on the patients. Importantly since these main hospitals were located in urban and semi-urban areas, those patients residing in the vicinity of these hospitals had better access to these hospitals than their rural counterparts. On the other hand, patients residing in the rural settings had the option to visit the nearest vision centers - a satellite primary eye care clinic established by the main hospital for the convenience of the patients living in far-off rural settings, to avail the post-operative care services. Despite these efforts to reach out to the patient to provide comprehensive eye care services, most of them did not visit the hospital to complete the full cycle of follow-up visits post-surgery. It is, therefore, recommended that appropriate steps and incentives should be taken in order to improve and encourage complete regular follow-up of patients to the hospital following surgery, especially those from the faroff rural areas as such follow-up visits could have beneficial psychological impacts as evident from these study findings.

The limitations of this study were that as most cataracts are bilateral, second eye surgery can be important in improving binocular vision and thereby greatly augmenting the VFQoL further. This study however, was confined to first-eye cataract surgery and its impact on VFQoL and the results must be interpreted keeping in mind this caveat. Secondly, this study employed the same field investigators to collect data during both the baseline and follow-up surveys. A tendency among patients to provide answers that would please the field investigator by overstating satisfaction with vision related quality of life therefore cannot be ruled out. There were also strengths. The study reported findings from the largest sample size of patients availing services at multiple private not-for-profit hospitals pan India. This was a large multisite longitudinal study using validated tools and examining cases before and after cataract surgery. The high response rates in completion of questionnaires and the random selection of study subjects are also strengths of this study.

\section{CONCLUSION}

In conclusion, the findings from this study quantify the gains six-months following first-eye cataract surgery in three areas: general function, psychosocial impact and visual function. These results highlight that regular and complete patient follow-up visits and prescription and provision of spectacles post-surgery significantly improved vision related general functions and ensued better psychosocial outcomes amongst patient operated for cataract.

\section{ACKNOWLEDGEMENTS}

The authors wish to thank the participants of this study and the staff of the partner hospitals for facilitating recruiting of the participants. The views expressed in this paper are those of the authors and do not necessarily reflect the views of the organisations that facilitated this study, or the institutions employing the authors. The MFV QoL Study Team contributed to the planning of the study logistics, data collection and interpretation, and the members of this Team other than the named authors include (in alphabetical order): Alphones B, Gangadhara Rao B, Jahirkhan Pathan, Lalthlamuana Pautu, Malagapu Damodar, Mariappan S, Moksidul Rohaman, Pranjal Daimary, Sandeep Ahire, Sindhu S, Sivasankar Rao D, Sumit Awasthi, Tarram Taggu and Vibhuji Patel.

\section{Funding: No funding sources \\ Conflict of interest: None declared \\ Ethical approval: Not required}

\section{REFERENCES}

1. World Health Organization (WHO). The Constitution of the World Health Organization. Chron World Health Organ. 1947;1:29-43.

2. World Health Organization (WHO). Consultation on the Development of Standards for Characterization of Vision Loss and Visual Functioning. Geneva: WHO; 2003.

3. Tseng VL, Chlebowski RT, Yu F, Cauley JA, Li W, Thomas F, et al. Association of Cataract Surgery With Mortality in Older Women:Findings from the Women's Health Initiative. JAMA Ophthalmol, 2017.

4. Bourne RRA, Flaxman SR, Braithwaite T, Cicinelli MV, Das A, Jonas JB, et al. Magnitude, temporal trends, and projections of the global prevalence of blindness and distance and near vision impairment:a 
systematic review and meta-analysis. The Lancet Global Health. 2017;5:e888-e897.

5. Pascolini D, Mariotti SP. Global estimates of visual impairment:2010. British J Ophthalmol 2012;96:614-8.

6. Murthy G, Gupta SK, John N, Vashist P. Current status of cataract blindness and Vision 2020:the right to sight initiative in India. Indian $\mathbf{J}$ Ophthalmol. 2008;56:489-94.

7. Finger RP. Cataracts in India: current situation, access, and barriers to services over time. Ophthalmic Epidemiol. 2007;14:112-8.

8. Polack S, Eusebio C, Fletcher A, Foster A, Kuper H. Visual impairment from cataract and health related quality of life:results from a case-control study in the Philippines. Ophthalmic Epidemiol. 2010;17:152-9.

9. Finger RP, Kupitz DG, Holz FG, Balasubramaniam B, Ramani RV, Lamoureux EL, et al. The impact of the severity of vision loss on vision-related quality of life in India: an evaluation of the IND-VFQ-33. Investigative Ophthalmol Visual Sci. 2011;52:6081-8.

10. Taylor HR, Katala S, Munoz B, Turner V. Increase in mortality associated with blindness in rural Africa. Bull World Health Organ. 1991;69:335-8.

11. Sengupta M, Agree EM. Gender and Disability Among Older Adults in North and South India:Differences Associated with Coresidence and Marriage. J Cross-Cultural Gerontol. 2002;17:31336.

12. Foley D, Chowdhury J. Poverty, Social Exclusion and the Politics of Disability:Care as a Social Good and the Expenditure of Social Capital in Chuadanga, Bangladesh. Social Policy Admin. 2007;41:372-85.

13. Baltussen R, Sylla M, Mariotti SP. Costeffectiveness analysis of cataract surgery:a global and regional analysis. Bull World Health Organ. 2004;82:338-45.

14. Murthy GVS, Jain BK, Shamanna BR, Subramanyam D. Improving cataract services in the Indian context. Community Eye Health. 2014;27(28):4-5.

15. Dandona L. Cataract surgery in very elderly patients. Outcome of cataract surgery is poor in developing countries. BMJ. 2001;323:455.

16. Dandona L, Dandona R, Anand R, Srinivas M, Rajashekar V. Outcome and number of cataract surgeries in India:policy issues for blindness control. Clin Exp Ophthalmol. 2003;31:23-31.

17. Steinberg EP, Tielsch JM, Schein OD, Javitt JC, Sharkey P, Cassard SD, et al. The VF-14:an index of functional impairment in patients with cataract. Arch Ophthalmol. 1994;112:630-8.

18. Bellan L. Why are patients with no visual symptoms on cataract waiting lists? Can J Ophthalmol. 2005;40:433-8.

19. Acosta-Rojas ER, Comas M, Sala M, Castells X. Association between visual impairment and patientreported visual disability at different stages of cataract surgery. Ophthalmic Epidemiol. 2006;13:299-307.

20. Datta S, Foss AJ, Grainge MJ, Gregson RM, Zaman A, Masud T, et al. The importance of acuity, stereopsis, and contrast sensitivity for health-related quality of life in elderly women with cataracts. Invest Ophthalmol Vis Sci. 2008;49:1-6.

21. Mangione CM, Lee PP, Gutierrez PR, Spritzer K, Berry S, Hays RD. Development of the 25-item national eye institute visual function questionnaire. Arch Ophthalmol. 2001;119:1050-8.

22. Gupta SK, Viswanath K, Thulasiraj RD, Murthy GVS, Lamping DL, Smith SC, et al. The development of the Indian vision function questionnaire:field testing and psychometric evaluation. Br J Ophthalmol. 2005;89(5):621-7.

23. Kurian E. PRISM: Measuring the impact of cataract surgery. International Agency for the Prevention of Blindness. 2017. Available at: https://www.iapb.org/ news/prism-measuring-impact-cataract-surgery/.

Accessed on 3 August 2017.

24. World Health Organisation (WHO). International statistical classification of diseases and related health problems (ICD-10). 10th revision. WHO, Geneva; 2016. Available at: http://apps.who.int/ classifications/icd10/browse/2016/en\#/H53-H54 Accessed on 6 December 2017.

25. WHO. Consultation on development of standards for characterization of vision loss and visual functioning. Geneva: World Health Organization, 2003.

26. Murthy GVS, Gupta SK, Thulasiraj RD, Viswanath $\mathrm{K}$, Donoghue EM, et al. The development of the Indian vision function questionnaire:questionnaire content. Br J Ophthalmol. 2005;89:498-503.

27. Kazis LE, Anderson JJ, Meenan RF. Effect sizes for interpreting changes in health status. Med Care 1989;27(3 Suppl):S178-89.

28. Polack S, Eusebio C, Mathenge W, Wadud Z, Mamunur AK, Fletcher A, et al. The impact of cataract surgery on health related quality of life in Kenya, the Philippines, and Bangladesh. Ophthalmic Epidemiol. 2010;17(6):387-99.

29. Lamoureux EL, Fenwick E, Pesudovs K, Tan D. The impact of cataract surgery on quality of life. Curr Opin Ophthalmol. 2011;22:19-27.

30. Groessl EJ, Liu L, Sklar M, Tally SR, Kaplan RM, Ganiats TG. Measuring the impact of cataract surgery on generic and vision-specific quality of life. Qual Life Res. 2013;22:1405-14.

31. Ivers RQ, Cumming $R$, Mitchell $P$. Visual impairment and falls in older adults:The Blue Mountains Eye Study. J Am Geriatr Soc. 1998;46:58-64.

32. Marmamula S, Khanna RC, Shekhar K, Rao GN. Outcomes of Cataract Surgery in Urban and Rural Population in the South Indian State of AndhraPradesh:Rapid Assessment of Visual Impairment (RAVI) Project. PLoS One. 2016;11(12):1. 
33. National Programme for Control of Blindness. Guidelines for voluntary and government organisations. 12th Five-year plan report. Directorate General of Health Services. Ministry of Health and Family Welfare, Government of India; New Delhi, 2014.
Cite this article as: Kurian E, Vishwakarma $\mathrm{P}$,

Deenadayalan J, Mondal A, Ranpise D, Chavan S, et al. Determinants of vision function related quality of life of patients undergoing unilateral cataract surgical services in charitable hospitals in India: a multicentre prospective cohort study. Int J Community Med Public Health 2018;5:1138-48. 